\title{
A Conceptual Study on Image Enhancement Techniques for Fingerprint Images
}

\author{
K. Krishna Prasad ${ }^{1} \&$ P. S. Aithal ${ }^{2}$ \\ ${ }^{1}$ Research Scholar, College of Computer and Information Science, Srinivas University, \\ Mangaluru-575001, Karnataka, India \\ ${ }^{2}$ College of Computer and Information Science, Srinivas University, Mangaluru-575001, \\ Karnataka, India \\ E-mail: karanikrishna@gmail.com
}

Type of the Paper: Research Paper.

Type of Review: Peer Reviewed.

Indexed In: OpenAIRE.

DOI: http://dx.doi.org/10.5281/zenodo.831678.

Google Scholar Citation: IJAEML

\section{How to Cite this Paper:}

Krishna Prasad, K., Aithal, P. S. (2017). A Conceptual Study on Image Enhancement Techniques for Fingerprint Images. International Journal of Applied Engineering and Management Letters (IJAEML), 1(1), 63-72.

DOI: http://dx.doi.org/10.5281/zenodo.831678.

International Journal of Applied Engineering and Management Letters (IJAEML)

A Refereed International Journal of Srinivas University, India.

(C) With Authors.

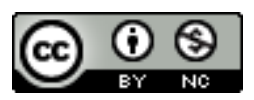

This work is licensed under a Creative Commons Attribution-Non Commercial 4.0 International License subject to proper citation to the publication source of the work.

Disclaimer: The scholarly papers as reviewed and published by the Srinivas Publications (S.P.), India are the views and opinions of their respective authors and are not the views or opinions of the S.P. The S.P. disclaims of any harm or loss caused due to the published content to any party.

K. Krishna Prasad et al, (2017); www.srinivaspublication.com 


\title{
A Conceptual Study on Image Enhancement Techniques for Fingerprint Images
}

\author{
K. Krishna Prasad ${ }^{1} \&$ P. S. Aithal ${ }^{2}$ \\ ${ }^{1}$ Research Scholar, College of Computer and Information Science, Srinivas University, \\ Mangaluru-575001, Karnataka, India \\ ${ }^{2}$ College of Computer and Information Science, Srinivas University, Mangaluru-575001, \\ Karnataka, India \\ E-mail: karanikrishna@gmail.com
}

\begin{abstract}
Biometrics is an emerging field of research in recent years and has been devoted to the identification of individuals using one or more intrinsic physical or behavioral traits. Fingerprints are the prominent and widely acceptable biometric features compared to face, speech, iris, and other types of biometrics. Fingerprint characteristic or features are unique for everyone and which cannot change throughout the lifetime. Fingerprint biometrics is having applications in diverse fields like attendance system, criminology, mobile applications and logical access control system. This is the purpose behind the popularity of fingerprints as the biometric identifier. The biometric image captured through mobile supportive devices like the mobile camera or USB Fingerprint contains low-quality images. In fingerprint recognition system the quality of the image plays a very important role while matching two fingerprints. Most of the fingerprint recognition systems result in poor matching due to impurity or noisy images. So there is high necessity and scope for image preprocessing and enhancement techniques in order to improve the quality of fingerprint image and to obtain high accuracy in the matching process. In this paper, we discuss some approaches and methods for reducing noise or impurities and to improve the quality of the image before matching them. These techniques help the fingerprint recognition system to become robust and to obtain high quality in the matching process.
\end{abstract}

Keywords: Fingerprint, Ridge, Pattern, Enhancement, Thinning, Binarisation, Filtering.

\section{INTRODUCTION :}

Biometrics is unique metrics related to human characteristics, which can be used for identification or authentication purpose as individual's claimed identity. Every human being can be recognized through observation of particular characteristics, which mainly involves different types as visual biometrics, chemical biometrics, auditory biometrics, behavioral biometrics, Olfactory or odor biometrics and spatial biometrics [1]. The qualities utilized for human distinguishing proof on the premise of their all inclusiveness, uniqueness, perpetual quality, quantifiability, and agreeableness. There are different biometric identifiers however fingerprints are the most broadly utilized among them. Fingerprints have two properties that are utilized for distinguishing proof:

i) Uniqueness: This property says that everybody has one of a kind fingerprint. No two people regardless of the possibility that they are twins can have same fingerprints. No two fingerprints on the same hand have ever been observed to be similar.The example of edges and valleys are distinctive for each person and for each finger.

ii) Permanence: This property says that the fingerprints of a man are changeless i.e. they don't change all through the lifetime of that individual. Regardless of the possibility that there is a scar on the finger of a man than the outline of the edges and valleys will recuperate after some time. 


\subsection{Definition of Fingerprint}

A fingerprint is the pattern of lines called ridges and some special structures on the surface of finger called furrows. Ridges are combinations of ridge flow, ridge characteristics and ridge structure, which is common elements in all fingerprints and ride characteristics are unique called minutiae.

\subsection{Features of Fingerprint}

Fingerprint identification is one of the most important biometric technologies compared to other biometrics due to its popularity and widely available technologies, which has drawn a considerable amount of interest recently [2-3]. The unique feature of the fingerprint is determined by the local ridge characteristics called minutiae, which are one of the most important criteria used in fingerprint recognition system [4]. There are more than 150 minutiae characteristics are identified in the literature. These local ridge characteristics are not equally distributed. Minutiae are classified into two types based on minutiae points as ridge ending and bifurcation. Ridge ending starts at a point and ends in another point suddenly [5]. Bifurcation is the feature in which ridge starts from an arbitrary point and moves in a path and at any, some other arbitrary point splits into two paths or simply ridge forks. A good quality fingerprint image comprises of at least 50-100 minutiae.

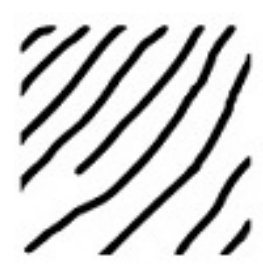

(a)

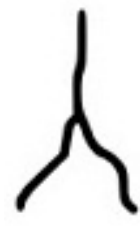

(b)

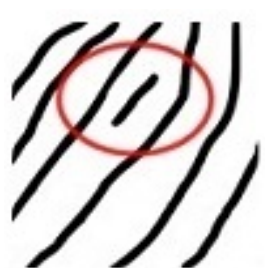

(c)

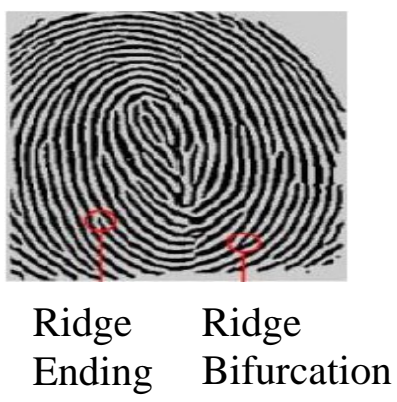

(d)

Figure 1: (a) Ridge ending (b) Bifurcation (c) Short Ridge (Dot) (d) Ridge ending and Ridge Bifurcation overlaid in fingerprint image [6]

Ridge ending and Ridge Bifurcation overlaid in fingerprint image Automatic fingerprint matching algorithm compares these local ridge characteristics (minutiae) and their relationship to obtain scores at the time of personal identification and verification. Two ridges are separated by low lines called valleys. The valleys and ridges are usually represented by white and black lines or colors in fingerprint images. There are another two characteristics of minutiae core and delta, which are used for matching of fingerprints. Core represents the center from which ridge ending and ridge bifurcation or simply pattern are made. Delta is the point on friction ridge, at or nearest to the point of divergence of ridge ending and ridge bifurcation and which looks like the shape of delta symbol. Delta is also pointed from which Loop pattern, Whorl Pattern, and Arch pattern deviate.

Loop pattern: Ridges enters from either side of the impression or pattern, re-curves or touches an imaginary line drawn from delta to the core and terminates on the same side from where it's originated. 


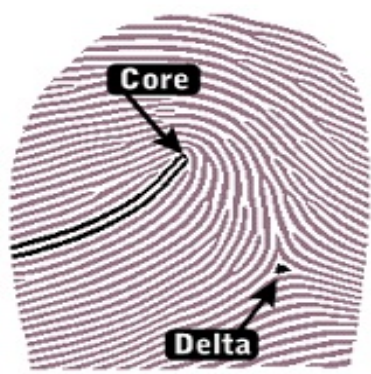

Figure 2: Core and delta of fingerprint image[7]

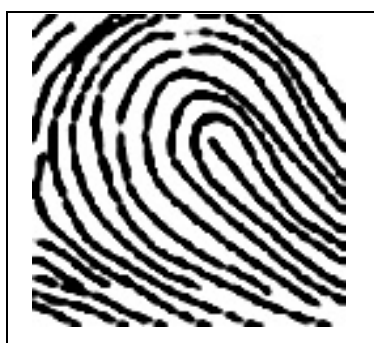

(a)

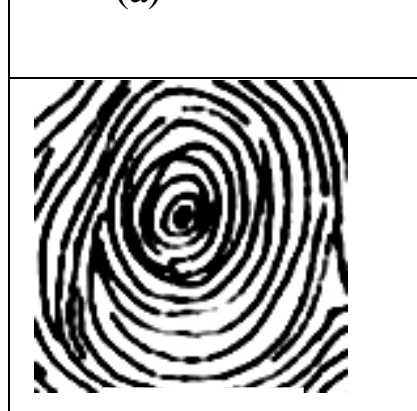

(e)

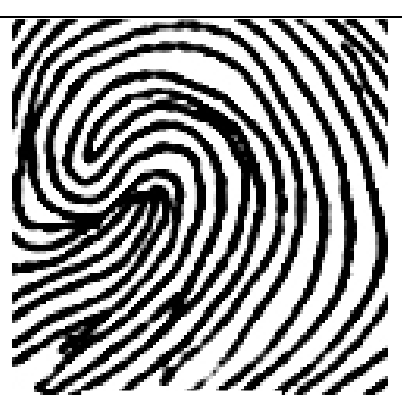

(i)

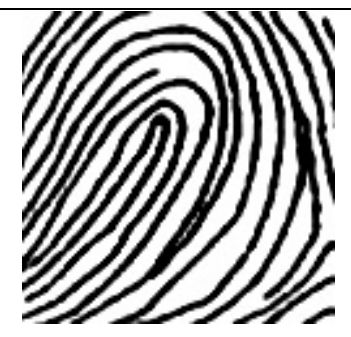

(b)

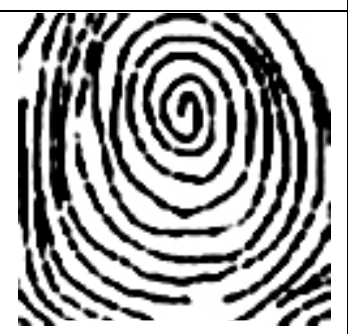

(f)

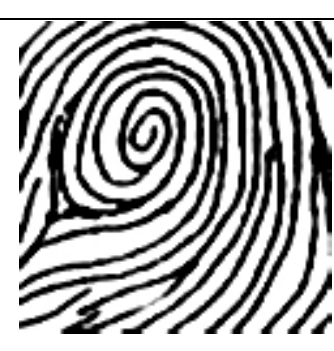

(j)

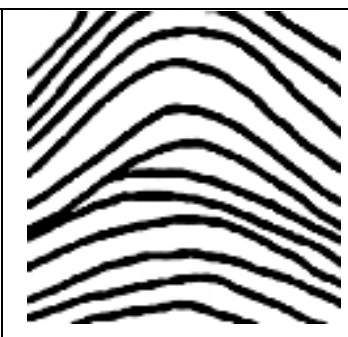

(c)

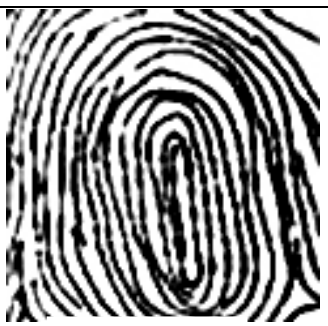

(g)

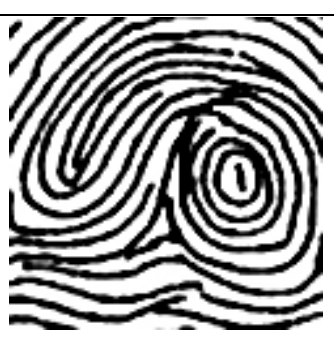

(k)

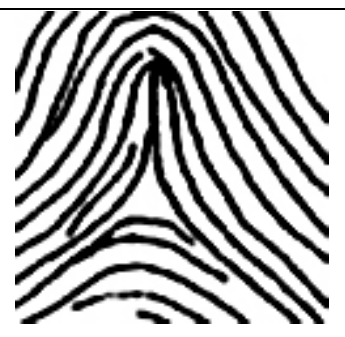

(d)

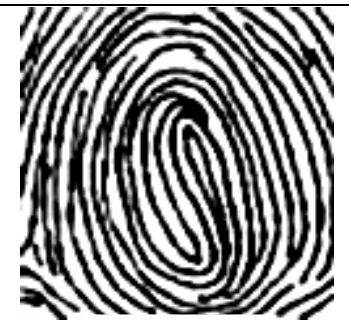

(h)

Figure 3: Basic patterns of fingerprints- (a) Ulnar Loop (b) Radial Loop (c) Simple Arch (d) Tented Arch (e) Concentric Whorl (f) Spiral Whorl pattern (g) Press Whorl (h) Imploding Whorl (i) Composite Whorl (j) Peacock's Eye (k) Variant pattern [8].

There are two types of Loop patterns; they are Ulnar Loop patterns and Radial Loop patterns. Ulnar Loop patterns are like a waterfall flowing towards the right with triangular points. Radial Loop patterns are opposite to Ulnar Loop patterns flowing towards left and shape is like a waterfall. In Arch, pattern ridges start from one side of the fingerprint pattern to another side without doing backward turn. Tented Arch is like a camping tent with sharp endings. Delta is not involved in Arch patterns. Whorl pattern consists of series of circles which starts from an arbitrary point and ends at the same point. In Concentric Whorl designs line begins from the focal point of the little circle, the lines 
on the fingertip gives off an impression of being little circles and spreads out like concentric circles with two triangle points. A spiral Whorl pattern consists of a winding model beginning from the inside and moves outward, has two triangular focuses. In Press Whorl patterns, like the whorl design, yet the circle transforms into a long oval shape, has two triangular focuses. Imploding Whorl patterns contains jujitsu or spiral like patterns in the center, encompassed by multi-layers of the circle. Composite Whorl patterns are similar to imploding Whorl without multi layered circle surrounding it. Peacock's Eye pattern from the inside would appear that a peacock's eyes and lips; the middle comprises of more than one circle or winding, the finish of each ring is associated in a straight line. It has two triangular focuses; one further and the other nearer to the inside. Variant pattern frequently has the mix of at least two of whorls, ulnar circles, or basic curves, with at least two triangle focuses.

\subsection{Fingerprint Recognition}

Fingerprint recognition system utilizes two types datasets called as training data set and test datasets. The training dataset is used for training purpose, initially, the fingerprint image is preprocessed and enhanced and later features are extracted and stored as a template. In test datasets, the same process is repeated but the template is not stored and just compared with the already stored template and matching score is calculated by utilizing an automated computer system. The stored features are compared for one to one match called verification and one-to many called as identification [9].

\section{RELATED WORK :}

L.Hong et al. [10] proposed a fast fingerprint enhancement algorithm, which depended on ridge orientation and frequency and through that, they can able to obtain more clear ridge and valley structure of the initial fingerprint image. They have evaluated the performance of fingerprint enhancement algorithm using goodness index and found that incorporating enhancement algorithm improves verification accuracy. Sharat S. Chikkerur et al. [11] proposed a new approach for fingerprint enhancement based on Short Time Fourier Transform (STFT) Analysis, mainly used for non-stationary properties. The algorithm evaluated all inherent features of the fingerprint image and found that performance of the algorithm improved slightly better. The Three inherent features are foreground region mask, local ridge orientation, and local frequency orientation. Sebastian et al. [12] proposed an algorithm for fingerprint minutiae based on CLAHE (Contrast Limited Adaptive Histogram Equalization). Their primary intention was to know the performance of combining Clip Limit, standard deviation, and sliding neighborhood, three techniques for the fingerprint enhancement. Through a simulated investigation, paper stimulates for developing thinning process and enhancement of the image. S.Greenberg et al. [13] compare the detection and analysis of minutiae through fingerprint image binarisation and direct extraction of minutiae from gray scale fingerprint images. They used Histogram equalization, Wiener filtering, and image binarisation as first method and unique anisotropic filter for direct gray scale enhancement as the second method in order to compare binarisation and direct extraction of minutiae from gray scale image techniques with an ultimate goal to achieve image enhancement. They found that both methods show significant improvements in terms of efficiency and execution time.

Y. He et al. [14] studied, analyzed and proposed a new algorithm based on orientation fields for image enhancement. In order to reduce noise and to obtain a high-quality image and to obtain better matching results, image enhancement and minutiae matching are two important steps in auto computer assisted fingerprint recognition system. D. K. Misra et al. [15] developed a method for fingerprint image enhancement based on Fourier cosine transform and matching of fingerprint image based on region and line structure that live between minutiae pairs.

Jianwei Yang et al. (2002) [16] improved the existing algorithm for fingerprint feature extraction by extracting minutiae directly from an original gray level image without undergoing steps of binarisation and thinning and obtained considerable better performance in efficiency. Jianwei Yang et al. (2003) [17] introduced novel filter design method for fingerprint image enhancement using Traditional Gabor Filter (TGF) of the invention to overcome drawbacks in image dependent parameter selection strategy. They have modified existing Traditional Gabor Filter as Modified Gabor Filter (MGF). Their algorithm achieved a remarkable advantage in preserving fingerprint image 
structure and in image enhancement consistency. C. Lee et al. (2006) [18] studied recognition of fingerprint image captured by a mobile camera. In segmentation of fingerprint regions from background images, they concentrated on three aspects which include texture, color, and size information. They proposed a robust regression method to overcome the drawbacks of gradient based filtering inability to remove outliers. In the pre-processing stage, they divided the fingerprint images into small blocks and verified blocks quality using good or bad quality regions. Their pre-processing algorithm and experimental results showed good performance compared to conventional ones.

\section{IMAGE ENHANCEMENT TECHNIQUES :}

Fingerprint image enhancement techniques mainly focus on reducing noises as much as possible in training datasets image or selected image and to obtain high scores in the matching process. The accuracy of the test datasets image directly depends on the quality of the image in training dataset. Because of this purpose, the good quality image is an essential and basic requirement for the improved results of matching. But this is not so easy or possible to obtain a good quality image almost all the time due to skin problems, scars on fingers. These wounds or cuts may end up with false minutiae results even in the good quality image. The error or noisy fingerprint image cannot able to provide all features at the time of feature extraction, either it may give wrong data or may not give required features only. Looking into all these aspects, fingerprint image pre-processing or enhancement becomes necessary and essential. There are many fingerprint enhancing techniques are available in the literature, an overview of these techniques are explained in this paper [19].

3. A. Histogram Modeling: The histogram of the fingerprint image represents, how often or number of times a gray level occurs in a group of total gray levels of the image. The output image produced by the histogram contains noisy in terms of intensity levels. Histogram equalization is a special technique to adjust intensity or brightness and to enhance the contrast of the image. With the help of histogram equalization, we can get a uniform or identical histogram for the output image. This technique is mainly used for improvement in contrast of an image by adjusting the individual gray level of the image. The results of the histogram are shown in figure 4.

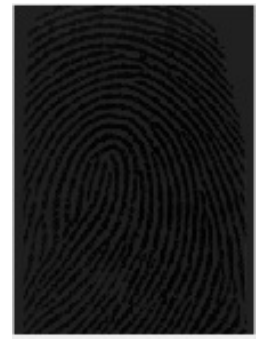

(a)

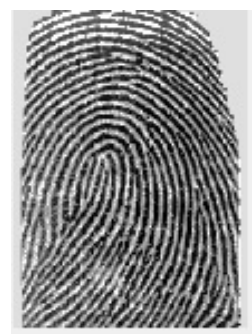

(c)

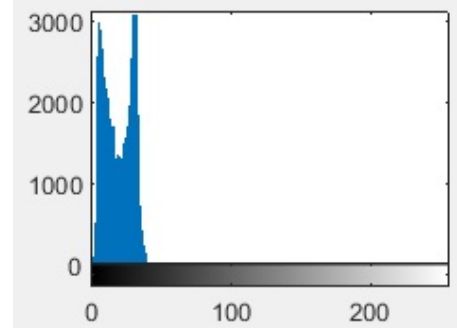

(b)

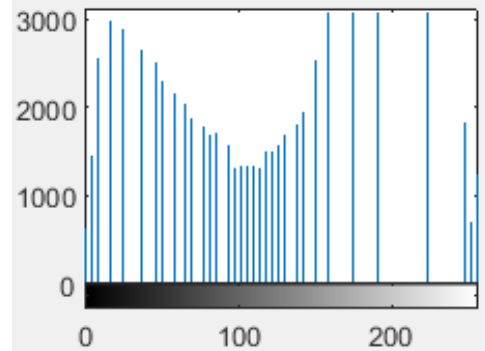

(d)

Figure 4: (a) Original image (b) Histogram of original image (c) Histogram equalized image (d)Histogram of equalized image

\section{B. Filtering Methods}

In fingerprint image recognition system, many filtering methods are available in the literature. These filtering methods ultimate purpose is to remove all types of error encountered in input or initial image and to improve the image recognition capacity in all aspects. Few methods are discussed below; 
Median Filtering: Median filtering considers statistical median of the pixels contained in a window around the pixel and it is a nonlinear filtering process used to remove impulsive noise and to enhance the fingerprint image quality. Median is calculated as follows

$\mathrm{V}(\mathrm{m}, \mathrm{n})=$ median $\{\mathrm{y}(\mathrm{m}-\mathrm{k}, \mathrm{n}-1),(\mathrm{k}, 1) \in \mathrm{W}\}$

Where $\mathrm{W}$ is the chosen Window. As like statistical figures, median filtering requires pixel values of the window should be arranged in order.

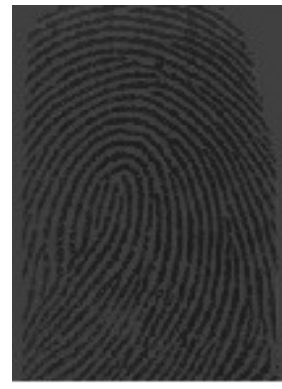

(a)

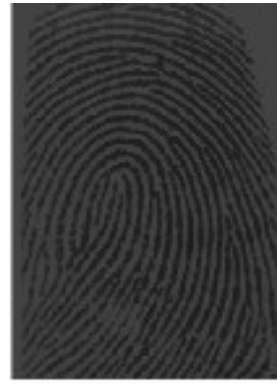

(b)

Figure 5: (a) Image before median filtering (b) Image after applying median filtering

High Pass filtering: High pass filtering is basically used to extract edges of the images. High pass filter helps to improve the quality of the image by sharpening the edges of the fingerprint image. Due to this reason, it is always good practice to do high pass filtering for the original image. The high pass filtering simply removes or the blurred image from the original image.

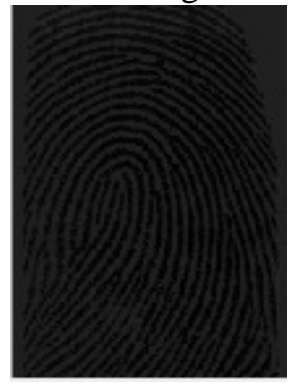

(a)

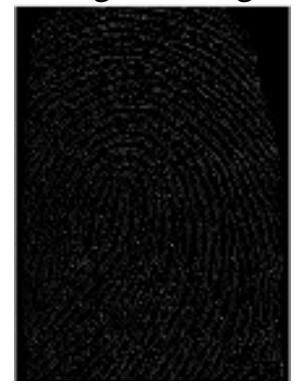

(b)

Figure 6: (a) Image before High pass filtering (b) Image after applying High pass filtering Weiner Filtering: Weiner filtering produces a good quality image by removing additive noises even when the image is having blur or low intensity. It minimizes maximum error in the process of noise smoothing, to improve the quality of the image.

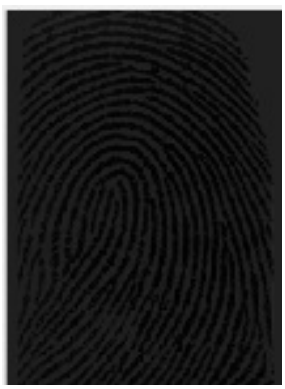

(a)

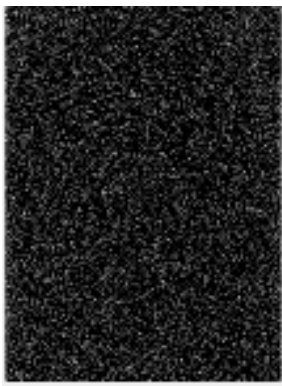

(b)

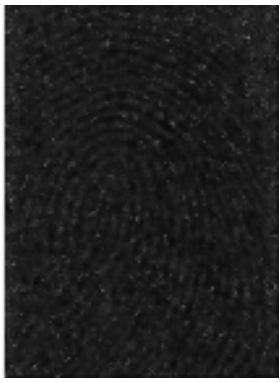

(c)

Figure 6: (a) Original image (b) Image after applying Noise (c) Image after applying Weiner Filter

Gabor Filtering: Gabor channel is a linear channel whose impulse reaction is characterized by a harmonic function and the result is multiplied by a Gaussian function. Gabor filter ideally catches both local orientation and frequency information from a fingerprint image. Once the ridge orientation and ridge frequency are calculated, at that point they are utilized to build the Gabor filter. In fingerprint enhancement, Gabor filter can be aligned to a particular frequency and orientation values. Gabor filter can improve the ridges towards local orientation. 


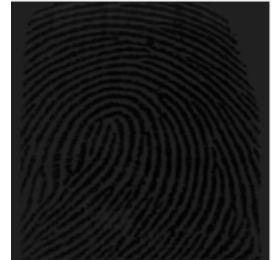

(a)

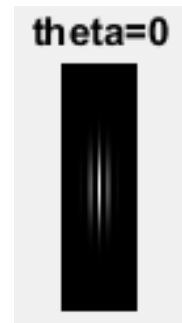

(b)

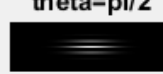

(f)

Figure 7: Gabor filtering size $7 \times 7$ and 4 orientations: -

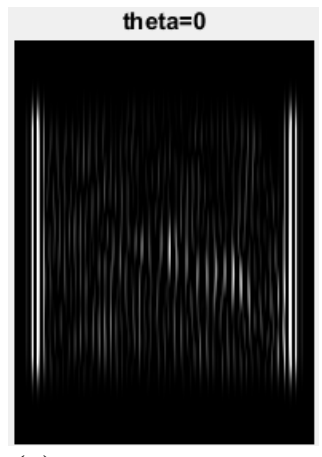

(c)

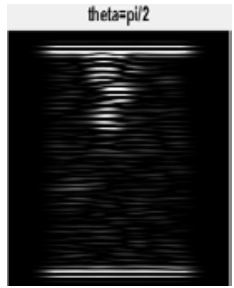

(g)

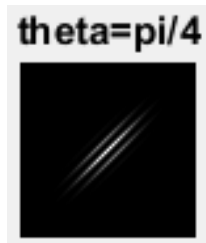

(d)

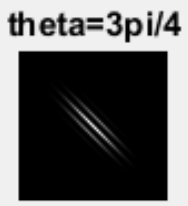

(h)

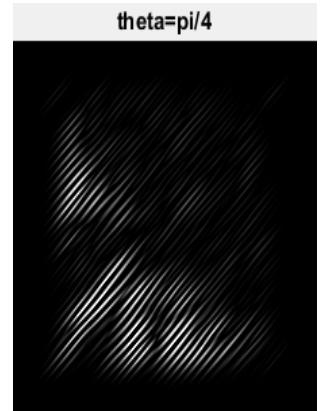

(e)

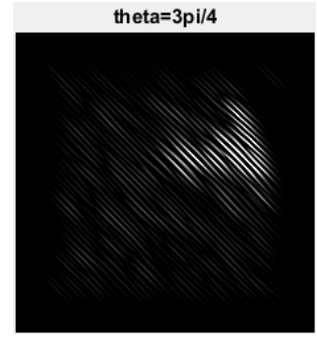

(i)

(a) Original Image

(b) orientation-theta $=0$ (c) orientation-theta $=\frac{\pi}{4}$ applied to original image (d) orientation-theta $=\frac{\pi}{4}$ (e) orientation-theta $=\frac{\pi}{4}$ applied to original image (f) orientation-theta $=\frac{\pi}{2}(e)$ orientation-theta $=\frac{\pi}{2}$ applied to original image (f) orientation-theta $=\frac{3 \pi}{4}(e)$ orientation-theta $=\frac{3 \pi}{4}$ applied to original

\section{C. Binarisation and Thinning} image

Binarisation is one of the preprocessing stages in automatic fingerprint recognition systems. If the input image is a color image, first it should be converted into gray scale image. From the gray scale image, a binary image is obtained by considering only two states as zero for ridges, which are represented by black color and one for the valley, which is represented by white color. In binarisation, we preset some threshold for pixels and pixel which is lower and higher than is threshold is represented by white and black color respectively.

Thinning is a special process that consecutively wears away the foreground pixels and finally produces lines that are almost one-pixel width. The first and foremost condition for thinning is input image should be a binary image and produces output as a binary image. Thinning is a final prior step to minutiae extraction in automatic fingerprint recognition system. Thinning is not achieved in a single step but it achieved through an iterative process. The connectivity of ridges and bifurcation can be reproduced from the thinning, means it preserves the basic structure of the image without affecting its original structure.

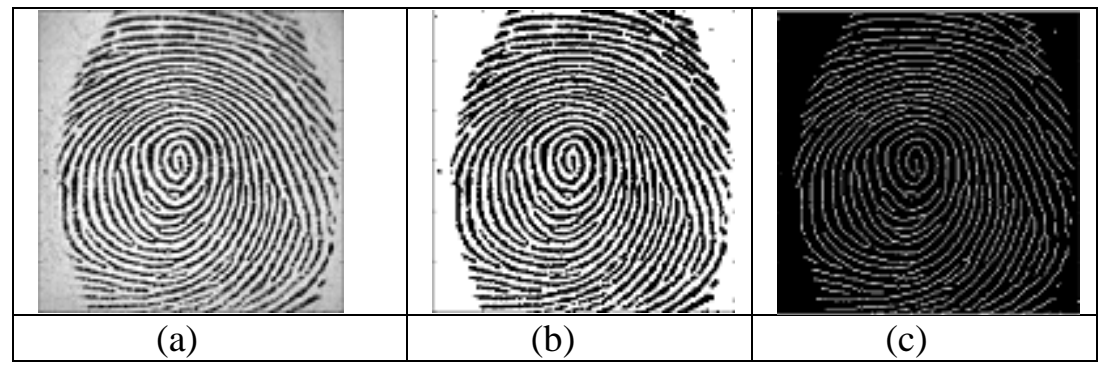

Figure 8: (a) Original image (b) Image after Binarisation (c) Thinning Image 


\section{CONCLUSION :}

In this paper, we have discussed basic concepts of fingerprint images and made a conceptual study on fingerprint image enhancement techniques. The diagram shown here are obtained by writing and running code in MATLAB. The fingerprint recognition system greatly affected by the quality of input image and if we apply image enhancement techniques on the input image, the noised can be reduced and also recognition system performance improved. Wish this paper could play an active role in image enhancement process in automatic fingerprint recognition system.

\section{REFERENCES :}

[1] Prabhakar, S., Pankanti, S., \& Jain, A. K. (2003). Biometric recognition: Security and privacy concerns. IEEE security \& privacy, 99(2), 33-42.

[2] Lee, H. C., Ramotowski, R., \&Gaensslen, R. E. (Eds.). (2001). Advances in fingerprint technology. CRC press.

[3] Newham, E. (1995). The biometric report. SJB services, 733.

[4] Moenssens, A. A. (1975). Fingerprint techniques. Chilton.

[5] Lee, C., Lee, S., Kim, J., \& Kim, S. J. (2006, January). Preprocessing of a fingerprint image captured with a mobile camera. In International Conference on Biometrics, Springer, Berlin, Heidelberg.348-355.

[6] https://images.google.com/. (2017). Google.[online] Available at: https://images.google.com/ridge ending and bifurcation images [Accessed 18 Jul. 2017].

[7] MVAY9O, I. A. P. R. Identification of Core and Delta Points in Fingerprint Images.

[8] Hong, L., Wan, Y., \& Jain, A. (1998). Fingerprint image enhancement: Algorithm and performance evaluation. IEEE transactions on pattern analysis and machine intelligence, 20(8), 777-789.

[9] Krishna Prasad, K. and Aithal, P. S.(2017). A Conceptual Study on User Identification and Verification Process Using Face Recognition Techniques. International Journal of Applied Engineering and Management Letters (IJAEML), (ISSN Applied), 1(1), 6-17. DOI:http://doi.org/10.5281/zenodo.810343.

[10] Hong, L., Wan, Y., \& Jain, A. (1998). Fingerprint image enhancement: Algorithm and performance evaluation. IEEE transactions on pattern analysis and machine intelligence, 20(8), 777-789.

[11] Chikkerur, S., Govindaraju, V., \& Cartwright, A. (2005). Fingerprint image enhancement using STFT analysis. Pattern Recognition and Image Analysis, 20-29.

[12] Sepasian, M., Balachandran, W., \& Mares, C. (2008, October). Image enhancement for fingerprint minutiae-based algorithms using CLAHE, standard deviation analysis and sliding neighborhood.In Proceedings of the World congress on Engineering and Computer Science 2224.

[13] Greenberg, S., Aladjem, M., Kogan, D., \&Dimitrov, I. (2000). Fingerprint image enhancement using filtering techniques. Proceedings of IEEE 15th International Conference on Pattern Recognition, 3, 322-325.

[14] He, Y., Tian, J., Luo, X., \& Zhang, T. (2003). Image enhancement and minutiae matching in fingerprint verification. Pattern recognition letters, 24(9), 1349-1360.

[15] Misra, D. K., Tripathi, S. P., \& Singh, A. (2012). Fingerprint image enhancement, thinning and matching. International Journal of Emerging Trends \& Tech in Comp Science (IJETTCS), 1(2), $17-21$. 
[16] Yang, J., Liu, L., Jiang, T., \& Fan, Y. (2002, August). An improved method for extraction of fingerprint features. In Proc. the 2nd Int. Conf. Image and Graphics, Anhui, PR China, 552-558.

[17] Yang, J., Liu, L., Jiang, T., \& Fan, Y. (2003). A modified Gabor filter design method for fingerprint image enhancement. Pattern Recognition Letters, 24(12), 1805-1817.

[18] Lee, C., Lee, S., Kim, J., \& Kim, S. J. (2006, January). Preprocessing of a fingerprint image captured with a mobile camera. In International Conference on Biometrics, Springer, Berlin, Heidelberg, 348-355.

[19] Saini, A. (2012). Image enhancement techniques for fingerprint images. International Journal of Emerging Trends and Technology in Computer Science, 1(3), 215-17. 